\title{
Current Density Impedance Imaging of an Anisotropic Conductivity in a Known Conformal Class
}

\author{
Nicholas Hoell $^{*} \quad$ Amir Moradifam ${ }^{\dagger} \quad$ Adrian Nachman ${ }^{\ddagger}$
}

February 28, 2013

\begin{abstract}
We present a procedure for recovering the conformal factor of an anisotropic conductivity matrix in a known conformal class in a domain in $\mathbb{R}^{n}$ with $n \geq 2$. The method requires one internal measurement, together with a priori knowledge of the conformal class (local orientation) of the conductivity matrix. This problem arises in the coupled-physics medical imaging modality of Current Density Impedance Imaging (CDII) and the assumptions on the data are suitable for measurements determinable from cross-property based couplings of the two imaging modalities CDII and Diffusion Tensor Imaging (DTI). We show that the corresponding electric potential is the unique solution of a constrained minimization problem with respect to a weighted total variation functional defined in terms of the physical data. Further, we show that the associated equipotential surfaces are area minimizing with respect to a Riemannian metric obtained from the data. The results are also extended to allow the presence of perfectly conducting and/or insulating inclusions.
\end{abstract}

Keywords: Anisotropic, Hybrid Problems, Conductivity, Diffusion Tensor Imaging, Current Density Impedance Imaging

\section{Introduction}

Biological tissues such as muscle or nerve fibres are known to be electrically anisotropic (see e.g. [35, 37]). In this paper, we consider the problem of recovering an anisotropic

${ }^{*}$ Department of Mathematics, University of Toronto, Toronto, Ontario, Canada M5S 2E4. E-mail: nhoell@math.toronto.edu. The author is supported in part by a MITACS Postdoctoral Fellowship.

${ }^{\dagger}$ Department of Applied Physics and Applied Mathematics, Columbia University, New York, NY, USA. E-mail: am3937@columbia.edu. The author is supported in part by Mitacs and NSERC Postdoctoral Fellowships.

‡Department of Mathematics and the Edward S. Rogers Sr. Department of Electrical and Computer Engineering, University of Toronto, Toronto, Ontario, Canada. E-mail: nachman@math.toronto.edu. The author is supported in part by an NSERC Discovery Grant. 
electric conductivity $\sigma$ of a body $\Omega$ from measurement of one current $J$ in the interior. Such interior data can be obtained by Current Density Imaging (CDI), a method pioneered at the University of Toronto ([17, 38]) that used a Magnetic Resonance Imager (MRI) in a novel way. We also rely on the MRI-based Diffusion Tensor Imaging (DTI) method to determine the conformal class of $\sigma$, as in the new DT-CD-II method recently introduced and tested experimentally in $([23])$. Thus, we assume that the matrix-valued conductivity function is of the form:

$$
\sigma(x)=c(x) \sigma_{0}(x),
$$

with $\sigma_{0}(x)$ known from DTI, and with the cross-property factor $c(x)$ a scalar function to be determined. This assumption is motivated by a number of physical studies which have shown a linear relationship between the conductivity tensor and the diffusion tensor (see e.g. [8, 23] and further references therein).

We show that, in dimension $n \geq 2$, the cross-property factor $c(x)$ can be determined from knowedge of the current $J$ in $\Omega$ and of the corresponding prescribed voltage $f$ on the boundary $\partial \Omega$. In fact, the only internal data we require is the scalar function

$$
a=\left(\sigma_{0}^{-1} J \cdot J\right)^{\frac{1}{2}}
$$

(with $\sigma_{0}^{-1}$ denoting the inverse of the matrix $\sigma_{0}$ ). This turns out to be the appropriate extension of the corresponding earlier result for isotropic conductivities ([32]), where the interior data was the magnitude $|J|$.

The method we will be presenting is based on the minimization of a weighted total variation functional defined in terms of $a(x)$ and $\sigma_{0}(x)$. (See Theorem 2.3 for the precise statement).

More generally, we will show that when $\Omega$ contains perfectly conducting and/or insulating inclusions, then knowledge of $a, \sigma_{0}$ and $f$ determines the location of these inclusions (except in exceptional cases), as well as the function $c(x)$ (hence also the anisotropic conductivity $\sigma)$ in their complement.

\subsection{Background and Motivation}

Mathematical work on non-invasive determination of internal conductivity has focused largely on the classical method of Electrical Impedance Tomography (EIT). There have been major advances in the understanding of this nonlinear inverse boundary value problem (see [40] for an excellent review; in particular, see [13, 18] for recent results on recovering anisotropic conductivities in a given conformal class for the special case of admissible manifolds). It has also been shown that the EIT problem is exponentially ill-posed, yielding images of low resolution (see [16, 25]).

In a new class of inverse problems (that includes the one studied here) one seeks to overcome the limitations of the reconstructions obtainable from classical boundary measurements by using data that can be measured noninvasively in the interior of the object. These are known in the literature as hybrid problems (also as coupled physics, interior data or 
multi-wave problems), as they couple two imaging modalities to obtain internal measurements. For overviews of such methods see [6, 20]. For imaging the electric conductivity, there are several approaches that combine aspects of EIT with MRI: MREIT, CDII, Electric Properties Imaging (see [34, 39] for recent reviews) or with ultrasound measurements: Acousto-Electrical Tomography ([41, 3, 21]), Impedance-Acoustic Tomography ([14]).

The starting point for the method presented here is the measurement of one applied current $J(x)$ at all points $x$ inside a region $\Omega$. We briefly recall the influential idea of [17, 38] for obtaining such interior measurements using MRI. The current $J$ induces a magnetic field $B(x)$. The component of $B$ parallel to the static field of the imager can be determined at any point inside $\Omega$ from the corresponding change in the phase of the measured magnetization at that location. By performing rotations of the object and repeating the experiment with the same applied current, all three components of $B$ can be recovered, and $J(x)$ is then computed using Ampére's law:

$$
J(x)=\frac{1}{\mu_{0}} \nabla \times B(x)
$$

where $\mu_{0}$ is the magnetic permeability (essentially constant in tissue). For our purposes, it is important to note that this Current Density Imaging (CDI) method works equally well in anisotropic media, as no knowledge of the conductivity is needed for the determination of the current density $J(x)$.

Inside the body being imaged the electric potential $u(x)$ corresponding to the voltage $f(x)$ on the boundary solves the following Dirichlet problem for the conductivity equation:

$$
\begin{aligned}
\nabla \cdot \sigma \nabla u & =0, \quad x \in \Omega \subset \mathbb{R}^{n} \\
\left.u\right|_{\partial \Omega} & =f
\end{aligned}
$$

where $\sigma$ is the (generally tensorial) conductivity of the material. In the case of isotropic conductivities, (i.e. scalar $\sigma$ ) considered in [34, 33, 32, 31, 29] and in the absence of insulating or perfectly conducting inclusions one can replace $\sigma$ in the above equation using Ohm's law $|J|=\sigma|\nabla u|$ to obtain the quasilinear, degenerate, elliptic, variable coefficient 1-Laplacian equation:

$$
\nabla \cdot\left(|J| \frac{\nabla u}{|\nabla u|}\right)=0, \quad x \in \Omega
$$

The above equation was first introduced, with the above derivation, in the article [19], where the Neumann problem was considered and examples of non-existence and non-uniqueness were given to explain that additional data was needed for determining the conductivity. In the article [31] it was shown that equipotential surfaces, namely the level sets of $u(x)$, are minimal surfaces with respect to the conformal metric $|J|^{\frac{2}{n-1}} I_{n}$, with $I_{n}$ the $n \times n$ identity matrix; this result was then used to treat the Cauchy problem for equation (4). The Dirichlet problem for equation (44) can also have infinitely many solutions (see [32]). A solution around this difficulty was found in [32], where the approach via the partial differential equation (44) 
was replaced by the study of the variational problem for which it is the Euler-Lagrange equation. It was shown that the solution of 3 is the unique minimizer for this problem. We recall these results in the following theorem.

Theorem 1.1. ([32] Let $\Omega \subset \mathbb{R}^{n}, n \geq 2$ be a domain with a connected $C^{1, \alpha}$ boundary, $\alpha>0$, and let $\mu$ denote Lebesgue measure on $\Omega$. Let $(f,|J|) \in C^{1, \alpha}(\partial \bar{\Omega}) \times C^{\alpha}(\Omega)$ with $|J| \neq 0$ $\mu$-a.e. be associated with an unknown conductivity $\sigma \in C^{\alpha}(\bar{\Omega})$. Then

$$
u_{\sigma}=\underset{v \in W^{1,1}(\Omega) \cap C(\bar{\Omega})}{\operatorname{argmin}}\left\{\int_{\Omega}|J||\nabla v| \mu(d x) ;\left.\quad v\right|_{\partial \Omega}=f \text { and } \mu(\{\nabla v=0\})=0\right\}
$$

exists and is unique.

Furthermore, $\sigma=\frac{|J|}{\left|\nabla u_{\sigma}\right|} \in L^{\infty}(\Omega)$ is the unique $C^{\alpha}(\bar{\Omega})$ scalar conductivity associated to the pair $(f,|J|)$.

A generalization of the above result was later obtained in the article [29], where the isotropic conductivity was shown to be determined from knowledge of $|J|$ on the complement of open regions on which $\sigma$ may be zero (in the case of insulating inclusions) or infinite (for perfectly conducting inclusions). We refer the interested reader to details in [29].

\section{$1.2 \quad$ Statement of Results}

In this article we will extend the imaging method described above to the case in which the conductivity is anisotropic and known to be of the form $\sigma(x)=c(x) \sigma_{0}(x)$ where $c(x)$ is an unknown scalar function and $\sigma_{0} \in C^{0}(\Omega, \operatorname{Mat}(\mathbb{R}, n))$ is a symmetric positive definite matrix-valued anisotropic term, assumed known.

We shall first prove an anisotropic analogue to Theorem 1.1 as a prelude to the more general results accounting for inclusions and less restrictive function spaces. For this, we will need to precisely define the class of data that arises from physical measurements.

Definition 1.2 (First notion of admissibility). A triplet $\left(f, \sigma_{0}, a\right) \in H^{\frac{1}{2}}(\partial \Omega) \times L_{+}^{\infty}(\Omega, \operatorname{Mat}(\mathbb{R}, n)) \times$ $L^{2}(\Omega)$ shall be said to be admissible if there exists a $c(x) \in L_{+}^{\infty}(\Omega)$ such that

$$
a=\left(\sigma_{0}^{-1} J \cdot J\right)^{\frac{1}{2}}
$$

where the current

$$
J=-c \sigma_{0} \nabla u
$$

corresponds to the potential $u \in H^{1}(\Omega)$, the weak solution to the BVP

$$
\left\{\begin{array}{l}
\nabla \cdot\left(c \sigma_{0} \nabla u\right)=0, \quad x \in \Omega \\
\left.u\right|_{\partial \Omega}=f .
\end{array}\right.
$$

The first theorem we prove concerns the minimization of the following functional:

$$
\mathcal{F}[v]:=\int_{\Omega} a\left(\sigma_{0} \nabla v \cdot \nabla v\right)^{\frac{1}{2}} d \mu
$$


where $\mu$ denotes the Lebesgue measure on $\Omega$. We present the following uniqueness result in section 2 .

Theorem 1.3. Let $\Omega \subset \mathbb{R}^{n}$ be a domain with a connected $C^{1, \alpha}$ boundary, $\alpha>0$, and let the triplet $\left(f, \sigma_{0}, a\right) \in C^{1, \alpha}(\partial \Omega) \times C^{\alpha}(\bar{\Omega}, \operatorname{Mat}(\mathbb{R}, n)) \times C^{\alpha}(\Omega)$ be an admissible triplet as in Definition 1.2 with $|J|>0$ Lebesgue - a.e. in $\Omega$. Denote by $\sigma=c \sigma_{0} \in C^{\alpha}(\bar{\Omega}, \operatorname{Mat}(\mathbb{R}, n))$ the unknown generating conductivity for this triplet. Then the following minimization problem

$$
\operatorname{argmin}\left\{\mathcal{F}[v] ; \quad v \in W^{1,1} \cap C(\bar{\Omega}), \mu\{\nabla v=0\}=0\right\}
$$

has a unique solution $u_{\sigma}$, where the functional $\mathcal{F}$ is as in (6) .

Furthermore, the unique $C^{\alpha}(\bar{\Omega}, \operatorname{Mat}(\mathbb{R}, n))$ conductivity generating the current density $J$ while maintaining the boundary voltage $f$ is given by $\sigma=c(x) \sigma_{0}(x)$ with the conformal factor c determined from the formula

$$
c=\frac{a}{\left(\sigma_{0} \nabla u_{\sigma} \cdot u_{\sigma}\right)^{\frac{1}{2}}} .
$$

Following this we establish, in the remainder of section 2 , the geometrical result that equipotential sets $u^{-1}(\lambda):=\{x ; u(x)=\lambda\} \cap \bar{\Omega}$ are in fact minimal surfaces with respect to a certain Riemannian metric on $\Omega$ which is defined in terms of of $\sigma_{0}(x)$ and $a(x)$.

After the above preliminary results, we prove the following main uniqueness result in section 4, which allows for inclusions and more general function spaces. The precise statement requires an appropriate notion of admissibility, formally defined in section 4 which involves some technical extensions of the criteria in Definition 1.2. It also requires an extension of the functional (6) appearing in Theorem [1.3. A full definition of the proper generalization is postponed until section 3 but it takes the form of a weighted total variation $\int_{\Omega}|D v|_{\phi}$, where the weight $\phi$ is defined in terms of $a$ and $\sigma_{0}$ and where $|D v|_{\phi}$ is a weighted distributional gradient discussed in section 3 .

Our uniqueness result also requires certain natural assumptions on the regions of perfect and zero conductance $O_{\infty}$ and $O_{0}$, respectively, as is discussed in greater detail in that section. Further, the zero set of the function $a$, denoted $S$, are also assumed to have a certain topological feature discussed in section 3 .

Theorem 1.4. Let $\Omega \subset \mathbb{R}^{n}, n \geq 2$, be a domain with connected Lipschitz boundary and let $\left(f, \sigma_{0}, a\right) \in H^{1 / 2}(\partial \Omega) \times C^{0}\left(\Omega \backslash\left(O_{\infty} \cup O_{0}\right), \operatorname{Mat}(\mathbb{R}, n)\right) \times L^{\infty}(\Omega)$ be an admissible triplet generated by an unknown conductivity $\sigma$ in the sense of Definition 4.1 . Then the potential $u$ is a minimizer of the problem

$$
u=\operatorname{argmin}\left\{\int_{\Omega}|D v|_{\phi}: v \in B V_{l o c}(\Omega \backslash \bar{S}) \text { and }\left.v\right|_{\partial \Omega}=f\right\},
$$

and if $\bar{u}$ is another minimizer of the above problem, then $\bar{u}=u$ in $\Omega \backslash\{|J|=0\}$. Consequently

$$
\sigma=\frac{a}{\left(\sigma_{0} \nabla u \cdot \nabla u\right)^{\frac{1}{2}}} \sigma_{0} \in C^{\alpha}(\Omega \backslash \bar{Z})
$$

is the unique conductivity generating the admissible data triplet $\left(f, \sigma_{0}, a\right)$ and $Z$ is an open set discussed later which accounts for the inclusions. 
With this shown, we lastly prove that level sets of such solutions as above minimize the area functional

$$
\mathcal{A}(\Sigma)=\int_{\Sigma} a d S
$$

over a suitable space of variations of subsets of $\Sigma \subset \Omega$.

An outline of this paper is as follows. In section 2 we present the main ideas building up to Theorem 2.3. Beginning with a formal statement of the problem, we describe the data of interest in our first definition of admissibility. We then proceed to establish the uniqueness result under this assumption. This section avoids the more technical details that arise from consideration of singular, perfectly conductive, and/or insulating inclusions and also considers potentials satisfying a more restrictive amount of smoothness. These more restrictive assumptions help clarify the main ideas that will be useful later. In section 3 we will introduce some tools from geometric measure theory needed to define an appropriate weighted space of bounded variation for our minimization problem. Most of the results presented in this section originated in the article [2]. In section 4 we formulate a more general notion of admissibility and present the proof of our main uniqueness result, Theorem 4.2. We then prove, in section 5, that equipotential sets minimize an area functional defined in terms of current density measurements. Finally, some of the technical facts on existence and uniqueness of solutions to a limiting form of the conductivity equation, suitable in the presence of inclusions with zero or infinite conductivity, as well as an equivalent optimization result in this setting, are briefly presented in section 6. Section 7 presents conclusions and acknowledgments.

\section{Anisotropic Current Density Impedance Imaging}

In this section we present a simplified exposition of the main results of this paper, in order to illustrate the basic ideas used in the argument and to motivate the more general results to be presented later. We also use this section to briefly introduce some of the key geometric measure-theoretic concepts we will need and expand upon later; some excellent references thereon may be found in [11, 12, 15, 24, 30].

\subsection{Uniqueness in the Variational Problem for Inversion}

Assume that the conductivity $\sigma$ is of the form $c(x) \sigma_{0}(x)$ with $c(x),\left(\sigma_{0}\right)_{i j}(x) \in C^{\alpha}(\bar{\Omega}), \alpha>0$, $c(x)>0$ and $\sigma_{0}$ symmetric and positive-definite throughout $\Omega$.

Throughout the paper we will be using the notation

$$
(\xi, \eta)_{\sigma_{0}}:=\left(\sigma_{0} \xi\right) \cdot \eta, \quad|\xi|_{\sigma_{0}}:=\left(\left(\sigma_{0} \xi\right) \cdot \xi\right)^{\frac{1}{2}}, \quad \xi, \eta \in C^{\alpha}\left(\bar{\Omega}, \mathbb{R}^{n}\right)
$$

to denote the inner product induced by $\sigma_{0}$, and the corresponding norm, where $\cdot$ will always be taken to denote the Euclidean dot product. We also define the space $W_{+}^{1,1}(\Omega)$ as

$$
W_{+}^{1,1}(\Omega):=\left\{v \in W^{1,1}(\Omega), \quad \mu(\{x, \nabla v=0\})=0\right\}
$$


In what follows $\nabla$ denotes the usual (i.e. non-covariant) partial differentiation and we use the Einstein summation convention over repeated upper/lower indices.

We begin by showing that the solution $u$ to the BVP (5) is a minimizer of an action on $\Omega$ that is defined in terms of the internal density magnitude $|J|_{\sigma_{0}^{-1}}$. This generalizes the corresponding result for isotropic conductivities in [32].

Lemma 2.1. Assume that $\left(f, \sigma_{0}, a\right)$ is an admissible triplet and let $u$ be a solution to the corresponding forward problem (5). Then $u$ is a minimizer of the action integral $\mathcal{F}[\cdot]$ defined by the following

$$
\mathcal{F}[v]:=\int_{\Omega} a(x)|\nabla v|_{\sigma_{0}} d x
$$

i.e. the relation

$$
\mathcal{F}[v] \geq \mathcal{F}[u]
$$

Holds for all $v \in W_{+}^{1,1}(\Omega)$ satisfying $\left.v\right|_{\partial \Omega}=f$.

Proof. Let $v \in W_{+}^{1,1}(\Omega)$. Since a comes from an admissible triplet, there is a choice of $c(x)$ such that $a(x)$ takes the form $a=|J|_{\sigma_{0}^{-1}}$. Then

$$
\begin{aligned}
\mathcal{F}[v] & =\int_{\Omega}|J|_{\sigma_{0}^{-1}}|\nabla v|_{\sigma_{0}} d \mu \\
& =\int_{\Omega} c(x)|\nabla u|_{\sigma_{0}}|\nabla v|_{\sigma_{0}} d \mu \\
& \geq \int_{\Omega} c(x)\left|(\nabla u, \nabla v)_{\sigma_{0}}\right| d \mu \\
& =\int_{\Omega} \sigma \nabla u \cdot \nabla v d \mu \\
& =\int_{\partial \Omega} f \sigma \frac{\partial u}{\partial \boldsymbol{n}} d S \\
& =-\int_{\partial \Omega} f J \cdot \boldsymbol{n} d S
\end{aligned}
$$

with $\boldsymbol{n}$ an outer-oriented normal to $\partial \Omega$ and where, in line (13), we have integrated by parts and applied the conductivity equation on $u$. Equality holds in line (12) if and only if $\nabla u$ and $\nabla v$ are parallel $\mu-$ a.e. In particular, we have

$$
\mathcal{F}[u]=-\int_{\partial \Omega} f J \cdot \boldsymbol{n} d S
$$

which, on comparing with the above, shows that $u$ is a minimizer, as claimed.

In order to prove the main result of this section we shall need to recall some basic notions from geometric measure theory. Firstly, by $\mathcal{H}^{d}(\Sigma)$ we denote the $d$-dimensional Hausdorff 
measure of a set $\Sigma \subset \Omega$ defined as

$$
\mathcal{H}^{d}(\Sigma):=\lim _{\delta \downarrow 0} \inf \left\{\sum_{j=1}^{n}\left(\operatorname{diam} E_{j}\right)^{d}, \quad \bigcup_{j \in \mathbb{N}} E_{j} \supset \Sigma, \quad \operatorname{diam} E_{j} \leq \delta\right\}
$$

The super-level set of a non-negative function $u(x) \in W^{1,1}(\Omega)$, given by $E_{t}:=\Omega \cap\{u>t\}$ has so-called locally finite perimeter, in the sense that the vector-valued Radon measure $\nabla \chi_{E_{t}}$ satisfies $\left|\nabla \chi_{E_{t}}\right|<\infty$ for almost all $t$. For such sets we shall be concerned with the reduced boundary.

Definition 2.2. The reduced boundary $\partial^{*} E$ of a set with locally finite perimeter is the set of points in $\mathbb{R}^{n}$ for which the following hold;

1. For all $\epsilon>0$ one has $\int_{B(x, \epsilon)}\left|\nabla \chi_{E}\right|>0$

2. The measure-theoretic outer normal $\nu(x)$ determined by

$$
v(x):=-\lim _{\epsilon \downarrow 0} \frac{\int_{B(x, \epsilon)} \nabla \chi_{E}}{\int_{B(x, \epsilon)}\left|\nabla \chi_{E}\right|}
$$

exists, and satisfies $|\nu(x)|=1$.

For a super-level set $E_{t}$ the unit normal $\nu_{t}(x)$ exists $\mathcal{H}^{n-1}-$ a.e $x \in \partial^{*} E_{t}$ (see the remarks in [32]).

We now present the main result of this section.

Theorem 2.3. Let $\Omega \subset \mathbb{R}^{n}, n \geq 2$, be a domain with a connected $C^{1, \alpha}$ boundary, $\alpha>0$, and let $\left(f, \sigma_{0},|J|_{\sigma_{0}^{-1}}\right) \in C^{1, \alpha}(\partial \Omega) \times C^{\alpha}(\bar{\Omega}, \operatorname{Mat}(\mathbb{R}, n)) \times C^{\alpha}(\Omega)$ be an admissible triple with $|J|>0 \mu-$ a.e. in $\Omega$. Denote by $\sigma \in C^{\alpha}(\bar{\Omega})$ the unknown generating conductivity for this triplet.

Then the following minimization problem

$$
\underset{\left.v\right|_{\partial \Omega}=f}{\operatorname{argmin}}\left\{\mathcal{F}[v] ; v \in W_{+}^{1,1} \cap C(\bar{\Omega})\right\}
$$

has a unique solution $u_{\sigma}$.

Further, the unique $C^{\alpha}(\bar{\Omega})$ conductivity generating the local current density $J$ while maintaing a boundary voltage $f$ is given by $\sigma=c(x) \sigma_{0}(x)$ with the conformal factor c determined by

$$
c(x)=\frac{|J|_{\sigma_{0}^{-1}}}{\left|\nabla u_{\sigma}\right|_{\sigma_{0}}}
$$


Proof. The proof is essentially the same as the proof of Theorem 1.1 given in [32] so we present a self-contained but tersely abbreviated proof and refer the reader to that article for full details. Note first that since the triplet is assumed admissible the previous lemma ensures that $\operatorname{argmin}_{\left.u\right|_{\partial \Omega}=f}\left\{\mathcal{F}[u] ; u \in W_{+}^{1,1} \cap C(\bar{\Omega})\right\}$ is nonempty. We choose a minimizer and call it $u_{\sigma}$.

To show uniqueness, assume to the contrary that another minimizer to problem 14, say $\tilde{u} \in W_{+}^{1,1} \cap C(\bar{\Omega})$, exists. Recalling the proof of Lemma 2.1 one sees the Cauchy inequality used in (12) ensures that $\nabla u_{\sigma}=\lambda(x) \nabla \tilde{u}$ for some non-negative $\lambda \mu-a . e$. From this it follows that

$$
\frac{\nabla u_{\sigma}}{\left|\nabla u_{\sigma}\right|}=\frac{\nabla \tilde{u}}{|\nabla \tilde{u}|}
$$

holds $\mu$-a.e. We show that this implies equality of the minimizers away from Lebesguenegligible sets.

In view of Lemma 2.2 of 32 , the above yields an identification of measure-theoretic normal vectors a.e.. It follows that the super-level set $E_{t}=\{\tilde{u}>t\} \cap \Omega$ has a measuretheoretic normal $\nu_{t}(x)=-\frac{\nabla \tilde{u}}{|\nabla \tilde{u}|}$ which is continuously extendible from the reduced boundary $\partial^{*} E_{t} \cap \Omega$ to the topological boundary $\partial E_{t} \cap \Omega$. It then follows from a result of De Giorgi (e.g. 4.11 in [15]) that, for almost all $t$, the region $\partial E_{t} \cap \Omega$ is a $C^{1}$-hypersurface with unit normal $\nu_{t}(x)$. From this, a parameterization of each connected component of this hypersurface shows that $u_{\sigma}$ remains constant on each such set.

We verify that, for each $t$ such that $\partial E_{t}$ is a $C^{1}$-hypersurface, each connected component $\Pi_{t}$ of $\partial E_{t}$ intersects $\partial \Omega$. Indeed, if not, then the Alexander duality theorem ([27]) implies that $\Pi_{t} \cup \partial \Omega$ admits a decomposition of the form $\Pi_{t} \cup \partial \Omega=O_{1} \cup O_{2} \cup\left(\mathbb{R}^{n} / \bar{\Omega}\right)$ with $O_{i}$ open and connected. We claim that $\left(\partial O_{1} \cup \partial O_{2}\right) \cap \Pi_{t} \neq \emptyset$. Indeed, were this not the case then $\partial O_{i} \cap \partial \Omega \neq \emptyset$ for $i=1,2$ and we could again apply the Alexander duality theorem to obtain the decomposition of $\mathbb{R}^{n} / \Pi_{t}$ into a union of two bounded and unbounded open, connected regions, $\Pi_{t}^{c}=O_{b} \cup O_{u}$. Since, by the contrary assumption $O_{1} \cup O_{2} \cup \Omega^{c}$ is connected and unbounded we have that $O_{1} \cup O_{2} \cup \Omega^{c} \subset O_{u}$ and thus, on taking complements, $O_{b} \subset\left(O_{1} \cup O_{2} \cup \Omega^{c}\right)^{c} \subset \Pi_{t}$. This contradiction shows that $\left(\partial O_{1} \cup \partial O_{2}\right) \cap \Pi_{t} \neq \emptyset$, as claimed. It thereby follows that $\Pi_{t} \cap \partial \Omega \neq \emptyset$. In other words, connected components of almost all the level sets $\partial E_{t}$ reach the boundary $\partial \Omega$.

Now define $G:=\left\{t \in \mathbb{R}_{+}:\left.\tilde{u}\right|_{\partial E_{t}}=\left.u_{\sigma}\right|_{\partial E_{t}}\right\} \subset \mathbb{R}_{+}$and suppose there exists a ball $B \subset \Omega$ whose closure is contained in $\Omega$ and such that $\bar{B} \cap\{\tilde{u} \in G\}=\emptyset$. If so then since $|\nabla \tilde{u}|_{B} \neq 0 \mu$-a.e., $\tilde{u}$ must map $\bar{B}$ to the closed interval $[a, b]$. However $\mu([a, b])>0$ whereas $\mu\left(G^{c} \cap \Omega\right)=0$, a contradiction. We conclude from this that the union of all preimages of $G$ under $u$ and $\tilde{u}$ is dense in $\bar{\Omega}$ and therefore $u=\tilde{u} \mu-a . e$., as was to be demonstrated.

Finally, with $J=-c \sigma_{0} \nabla u_{\sigma}$ we have $\left(\sigma_{0}^{-1} J \cdot J\right)^{\frac{1}{2}}=\left(c^{2} \sigma_{0} \nabla u_{\sigma} \cdot \nabla u_{\sigma}\right)^{\frac{1}{2}} \mu-a . e$. This gives the desired formula for $c(x)$. 


\subsection{Equipotential Sets are Minimal Surfaces in a Riemannian met- ric Determined from the Data}

We close this section with some interesting geometrical results about the level sets of solutions to (5). Given $\sigma_{0}$ and the magnitude $|J|_{\sigma_{0}^{-1}}$ of the current, we define a Riemannian metric on $\Omega$ and show that the level sets of the corresponding potential function have zero mean curvature in this metric. In section 5 we will prove the stronger statement that these equipotential sets are in fact area minimizing. These are generalizations to anisotropic conductivites of results proved in [33, 31] for the isotropic case.

As is customary, we denote $|A|:=\operatorname{det} A$ for $A \in \operatorname{Mat}(\mathbb{R}, n)$ (which should not be mistaken for the norm $|V|_{\sigma_{0}}$ of a vector field $V$, as we hope shall be clear from the context).

Proposition 2.4. Let $\Omega \subset \mathbb{R}^{n}, n \geq 2$ be a domain with Lipschitz boundary and $u \in C^{1, \alpha}(\bar{\Omega})$, $\alpha>0$. Assume the conductivity $\sigma$ is of the form $c(x) \sigma_{0}(x)$ for $c, \sigma_{0} \in C^{\alpha}(\Omega)$ with $\sigma_{0}$ a known positive-definite matrix-valued function and that $|\nabla u|, c(x)>0 \mu$-a.e. where $u$ is the potential corresponding to the conductivity $\sigma$ and current density $J$ via $J=-\sigma \nabla u$.

Define the following Riemannian metric $g_{i j}$ on $\Omega$ :

$$
g_{i j}:=\left(\left|\sigma_{0}\right||J|_{\sigma_{0}^{-1}}^{2}\right)^{\frac{1}{n-1}}\left(\sigma_{0}^{-1}\right)_{i j}
$$

Then inside $\Omega$ one has that

$$
\nabla \cdot\left(\sqrt{|g|} \frac{g^{i j} \nabla_{i} u}{\left\|g^{-1} \nabla u\right\|_{g}}\right)=0
$$

Proof. We begin by noticing that $\left|\sigma_{0}\right|^{\frac{1}{n-1}}|J|_{\sigma_{0}^{-1}}^{\frac{2}{n-1}} \sigma_{0}^{-1}=c^{1+\frac{1}{n-1}-\frac{n}{n-1}}\{|\sigma|(\sigma \nabla u \cdot \nabla u)\}^{\frac{1}{n-1}} \sigma^{-1}$ whereby, with the above choice of $g_{i j}$ one has that

$$
g^{-1}=\{|\sigma|(\sigma \nabla u \cdot \nabla u)\}^{\frac{1}{1-n}} \sigma
$$

Defining $m(x):=|\sigma|(\sigma \nabla u \cdot \nabla u)$ it immediately follows that $|g|=\frac{m^{\frac{n}{n-1}}}{|\sigma|}$. Since $\left\|g^{-1} \nabla u\right\|_{g}^{2}=$ $\left\{\left(g^{-1} \nabla u\right) \cdot g\left(g^{-1} \nabla u\right)\right\}^{2}$ we have $\left\|g^{-1} \nabla u\right\|_{g}=\sqrt{\left(g^{-1} \nabla u\right) \cdot \nabla u}$. Then

$$
\begin{aligned}
\nabla \cdot\left(\sqrt{|g|} \frac{g^{i j} \nabla_{i} u}{\left\|g^{-1} \nabla u\right\|_{g}}\right) & =\nabla \cdot\left(\frac{m^{\frac{n+1}{2(n-1)}-\frac{1}{n-1}} \sigma \nabla u}{\sqrt{|\sigma| \sigma \nabla u \cdot \nabla u}}\right) \\
& =\nabla \cdot\left(\frac{\sqrt{m(x)} \sigma \nabla u}{\sqrt{m(x)}}\right)
\end{aligned}
$$

It follows from the fact that $u$ solves the conductivity equation that

$$
\nabla \cdot\left(\sqrt{|g|} \frac{g^{i j} \nabla_{i} u}{\left\|g^{-1} \nabla u\right\|_{g}}\right)=0 .
$$


The above result immediately implies the following.

Corollary 2.5. Suppose that $u, c, \sigma_{0}$ are as is in proposition (2.4). Then the level sets of $u$, $u^{-1}(\lambda):=\{u=\lambda\} \cap \bar{\Omega}$ are surfaces of zero mean curvature in the metric

$$
g_{i j}=\left(\left|\sigma_{0}\right||J|_{\sigma_{0}^{-1}}^{2}\right)^{\frac{1}{n-1}}\left(\sigma_{0}^{-1}\right)_{i j}
$$

Proof. As in the preceding proof of Theorem 2.3 the level sets $u^{-1}(\lambda)$ are $C^{1}$-hypersurfaces for $\mu$-a.e. $\lambda$. The unit vector $\eta:=\frac{g^{-1} \nabla u}{|\nabla u|_{g}}$ is $g$-orthogonal to each such level set $u^{-1}(\lambda)$ since if $\xi \in T_{x} u^{-1}(\lambda)$ then $\left.\xi \cdot \nabla u\right|_{x \in u^{-1}(\lambda)}=0$ and therefore

$$
\begin{aligned}
g(\xi, \eta) & =\frac{g\left(\xi, g^{-1} \nabla u\right)}{|\nabla u|_{g}} \\
& =\frac{\xi \cdot \nabla u}{|\nabla u|_{g}} \\
& =0
\end{aligned}
$$

Thus $\pm \eta$ are unit normals to the hypersurfaces $u^{-1}(\lambda)$ in the $g_{i j}$ metric. Since the mean curvature of a surface with unit normal $n$ is $H=\operatorname{div}_{g}(n)$, with $\operatorname{div}_{g}$ being the metric divergence, we conclude from Proposition (2.4) that when $u$ satisfies the conductivity equation, we have $H=0$.

\section{Preliminaries for the General Case}

In this section we prepare to expand upon the results in the preceding section by considering the conductivity equation over domains which may contain insulating or perfectly conducting inclusions, i.e. regions of infinite or zero conductivity, respectively. We shall give the appropriate reformulation of the forward problem (5) in this setting. We also discuss integration by parts and coarea formulae for spaces of bounded weighted variation which will play a key role in our main general uniqueness result.

\subsection{Weighted Total Variation}

We start by presenting some needed preliminary results about functions of bounded weighted variation. We will always use the notation $\chi_{A}(x)$ to denote the characteristic function of a set $A$. Often, we will abbreviate vectors and matrices in component form, in addition, as earlier, we will employ the Einstein summation convention of implied summation over repeated upper and lower indices wherever appropriate. 
Let $\Omega \subset \mathbb{R}^{n}$ be a bounded open set with connected Lipschitz boundary and let $a \in L^{\infty}(\Omega)$ be non-negative. While the function $a$ is now allowed to vanish, we require that its zero set $S:=\{x \in \bar{\Omega}: a(x)=0\}$ always be assumed to satisfy the following structural hypothesis

$$
S:=O \cup \Gamma
$$

where $\Gamma$ is a set of measure zero with at most countably many connected components, $\mathcal{H}^{n-1}(\partial \Omega \cap S)=0$, and where $O$ is a mutually disjoint union of finitely many $C^{1}$-diffeomorphic images of the unit ball, possibly empty. by

We recall the space of functions of locally bounded variation on a subset $\Sigma \subset \mathbb{R}^{n}$ is given

$$
B V_{l o c}(\Sigma):=\left\{u \in \operatorname{Lip}(\Sigma), \int_{K}|D u|<\infty, \quad \forall K \subset \Sigma, \quad K \text { compact }\right\}
$$

where $D u$ is the distributional gradient of $u$. This generalizes the space $B V(\Omega)$, the space of all $L^{1}(\Omega)$ functions with bounded total variation of the distributional gradient, i.e. those functions satisfying

$$
\int_{\Omega}|D u|<\infty
$$

Let $\sigma_{0} \in C^{0}(\Omega, \operatorname{Mat}(\mathbb{R}, n))$ be a symmetric positive definite matrix with components $\left(\sigma_{0}\right)_{i j}$ satisfying

$$
m|\xi|^{2} \leq \sum_{i, j=1}^{n}\left(\sigma_{0}\right)_{i j}(x) \xi^{i} \xi^{j} \leq M|\xi|^{2} \quad \forall x \in \Omega \backslash S, \quad \forall \xi \in \mathbb{R}^{n}
$$

for constants $0<m, M<\infty$. We then denote by $\phi(x, \xi)$ the following function

$$
\varphi(x, \xi)=a(x)\left(\sum_{i, j=1}^{n}\left(\sigma_{0}\right)_{i j} \xi^{i} \xi^{j}\right)^{\frac{1}{2}}
$$

For $u \in B V_{l o c}(\Omega \backslash S)$ we define the weighted total variation of $u$, with respect to $\varphi$, in $\Omega$ as

$$
\int_{\Omega}|D u|_{\varphi}=\sup _{B \in \mathfrak{B}_{a}} \int_{\Omega} u \nabla \cdot B d \mu
$$

where

$$
\mathfrak{B}_{a}=\left\{B \in L_{c}^{\infty}\left(\Omega, \mathbb{R}^{n}\right): \quad \nabla \cdot B \in L^{n}(\Omega) \quad \text { and } \quad|B|_{\sigma_{0}^{-1}} \leq a(x) \quad \text { a.e. in } \quad \Omega\right\} .
$$

and $L_{c}^{\infty}\left(\Omega, \mathbb{R}^{n}\right)$ is the space of vector fields of compact support in $\Omega$ whose components are in $L^{\infty}(\Omega)$.

It is a straightforward consequence of the definition (18) that $\int_{\Omega}|D u|_{\varphi}$ is $L_{l o c}^{\frac{n}{n-1}}(\Omega)$-lower semi-continuous. It was shown in [2] by Amar and Bellettini that for any $u \in B V(\Omega)$, one 
has the following integral representation formula for the weighted total variation appearing in equation (18),

$$
\int_{\Omega}|D u|_{\varphi}=\int_{\Omega} h\left(x, v^{u}\right)|D u|
$$

where, in the above,

$$
h\left(x, v^{u}\right):=\left(|D u|-\operatorname{ess} \sup _{\mathbf{B} \in \mathfrak{B}}\left(B \cdot v^{u}\right)\right)(x) \quad|D u|-\text { a.e. } x \in \Omega,
$$

and $v^{u}$ denotes the vectorial Radon-Nikodym derivative $v^{u}(x)=\frac{d D u}{d|D u|}$. Note that the righthand side of equation (19) makes sense, as $v^{u}$ is $|D u|$-measurable, and hence $h\left(x, v^{u}(x)\right)$ is as well. In particular, it can be shown (viz. 22 Prop. 7.1) that if $a$ and $\sigma_{0}$ are continuous in $\Omega$, then one has

$$
h\left(x, v^{u}\right)=a(x)\left(\sum_{i, j=1}^{n} \sigma_{0}^{i j} v_{i}^{u} v_{j}^{u}\right)^{1 / 2}, \quad|D u|-\text { a.e. in } \Omega
$$

for every Borel set $\Omega$ and $u \in B V(\Omega)$.

Following [1] and [5], we let

$$
X:=\left\{B \in L^{\infty}\left(\Omega, \mathbb{R}^{n}\right): \operatorname{div} B \in L^{n}(\Omega)\right\} .
$$

As proven in [5], Theorem 1.2, if $\nu_{\Omega}$ denotes the outer unit normal vector to $\partial \Omega$, then for every $B \in X$ there exists a unique function $\left[B \cdot \nu_{\Omega}\right] \in L_{\mathcal{H}^{n-1}}^{\infty}(\partial \Omega)$ such that

$$
\int_{\partial \Omega}\left[B \cdot v_{\Omega}\right] u d \mathcal{H}^{n-1}=\int_{\Omega} u \nabla \cdot B d \mu+\int_{\Omega} B \cdot \nabla u d \mu, \quad \forall u \in C^{1}(\bar{\Omega}) .
$$

Moreover, for $u \in B V(\Omega)$ each such $B \in L^{\infty}\left(\Omega, \mathbb{R}^{n}\right)$ with $\nabla \cdot B \in L^{n}(\Omega)$ gives rise to a Radon measure on $\Omega$, denoted $(B \cdot D u)$, satisfying the following

$$
\int_{\partial \Omega}\left[B \cdot v_{\Omega}\right] u d \mathcal{H}^{n-1}=\int_{\Omega} u \nabla \cdot B d \mu+\int_{\Omega}(B \cdot D u), \quad \forall u \in B V(\Omega),
$$

We refer the interested reader to [1, 5 ] for a proof.

We shall need the following lemma, a proof of which follows from (23), and the fact that

$$
B V_{l o c}(\Omega \backslash \bar{S}) \cap L^{\infty}(\Omega) \subset B V(\Omega)
$$

which can be easily verified.

Lemma 3.1. Let $S$ be as defined in (16)). Then

$$
\int_{\partial \Omega}\left[B \cdot v_{\Omega}\right] u d \mathcal{H}^{n-1}=\int_{\Omega} u \nabla \cdot B d \mu+\int_{\Omega}(B \cdot D u)
$$

for all $u \in B V_{l o c}(\Omega \backslash \bar{S}) \cap L^{\infty}(\Omega)$. 
We conclude with a useful co-area formula for functions of bounded weighted total variation. Details can be found in [2].

Theorem 3.2 (Generalized Co-Area Formula). Let $u \in B V(\Omega)$ and suppose $\mathcal{H}^{n-1}(\Omega \cap\{u=$ $s\})<\infty$ holds for all $s \in \mathbb{R}$. Let $P_{\phi}(A, \Omega)$ denote the perimeter of the set $A \subset \Omega$ given by

$$
P_{\phi}(A, \Omega):=\int_{\Omega}\left|D \chi_{A}\right|_{\phi}
$$

Then

$$
\int_{\Omega}|D u|_{\phi}=\int_{\mathbb{R}} P_{\phi}(\{u>s\}, \Omega) d s
$$

We note that this may, on using the representation formula (19), be recast as

$$
\int_{\Omega}|D u|_{\phi}=\int_{\mathbb{R}} \int_{\Omega \cap \partial^{*}\{u(x)>s\}} h\left(x, v^{s}\right) d \mathcal{H}^{n-1}(x) d s
$$

where $v^{s}$ is a unit outer-oriented normal vector to $\Omega \cap \partial^{*}\{u(x)>s\}$.

\subsection{Modeling Regions with Zero or Infinite Conductivity}

Here we discuss how to formulate a suitable version of the conductivity equation (5) in the presence of inclusions of infinite and/or zero conductivity.

Let $O_{\infty}$ be an open subset of $\Omega$ satisfying $\bar{O}_{\infty} \subset \Omega$, meant to model perfectly conducting inclusions, and $O_{0}$ be an open subset of $\Omega$ with $\bar{O}_{0} \subset \Omega$, meant to model insulating inclusions. We assume $\bar{O}_{\infty} \cap \bar{O}_{0}=\emptyset, \Omega \backslash \overline{O_{\infty} \cup O_{0}}$ is connected, and the boundaries $\partial O_{\infty}, \partial O_{0}$ are piecewise $C^{1, \alpha}$ for $\alpha>0$. We also assume that $O_{0}$ is a mutually disjoint union of finitely many

$C^{1}$ - diffeomorphic images of the unit ball, possibly empty. In addition, in two dimensions we require that $O_{0}$ has at most one such component.

Let $\sigma^{j k}$ and $\tilde{\sigma}^{j k}$ be symmetric positive definite matrix functions. For $k>0$ consider the conductivity problem

$$
\left\{\begin{array}{l}
\partial_{x_{j}}\left\{\left[\left(k \tilde{\sigma}^{i j}-\sigma^{i j}\right) \chi_{O_{\infty}}+\sigma^{i j}\right] \partial_{x_{i}} u_{k}\right\}=0, \text { in } \Omega \backslash \bar{O}_{0} \\
\frac{\partial u_{k}}{\partial \nu_{u}}=0 \text { on } \partial O_{0} \\
\left.u_{k}\right|_{\partial \Omega}=f .
\end{array}\right.
$$

The perfectly conducting inclusions occur in the limiting case $k \rightarrow \infty$. The limiting solution is the unique solution to the problem:

$$
\begin{cases}\partial_{x_{j}}\left(\sigma^{i j} \partial_{x_{i}} u\right)=0, & \text { in } \Omega \backslash \overline{O_{0} \cup O_{\infty}} \\ \nabla u=0, & \text { in } O_{\infty} \\ \left.u\right|_{+}=\left.u\right|_{-}, & \text {on } \partial\left(O_{0} \cup O_{\infty}\right) \\ \left.\int_{\partial O_{\infty}^{n}} \sigma \frac{\partial u}{\partial \nu_{n}}\right|_{+} d S=0, & n=1,2, \ldots \\ \left.\frac{\partial u}{\partial u}\right|_{+}=0, & \text { on } \partial O_{0} \\ \left.u\right|_{\partial \Omega}=f, & \end{cases}
$$


(see the Appendix for more details), where $O_{\infty}=\cup_{n=1}^{\infty} O_{\infty}^{n}$ is a partition of $O_{\infty}$ into connected components. Here, as in the rest of the paper, $\nu$ is the outward unit normal vector and the subscripts \pm indicate the limit taken from the outside and inside the domain, respectively.

Remark For Lipschitz continuous conductivities in any dimension $n \geq 2$, or for essentially bounded conductivities in two dimensions, the solutions of the conductivity equation satisfy the unique continuation property (see, 9] and references therein). Consequently the insulated (and possibly perfectly conducting) inclusions are the only open sets on which the interior data $|J|_{\sigma_{0}^{-1}}$ may vanish identically. However, in three dimensions or higher it is possible to have a Hölder continuous $\sigma$ and boundary data $f$ that yield $u \equiv$ constant in a proper open subset $O_{s} \subsetneq \Omega$, see [36, 26]. We call such regions $O_{s}$ singular inclusions. On the other hand, we will not use Ohm's law in the classical sense inside perfect conductors: the current $J$ inside perfectly conducting inclusions is not necessarily zero whereas $\nabla u \equiv 0$ within such regions (see [4, 22]).

\section{Uniqueness and Determining the Conformal Class}

From now on we assume that $\sigma \in C^{\alpha}(\Omega, \operatorname{Mat}(\mathbb{R}, n))$ for $\alpha>0$ and satisfies

$$
\sigma(x)=c(x) \sigma_{0}(x)
$$

where $c(x) \in L_{+}^{\infty}\left(\Omega \backslash\left(O_{\infty} \cup O_{0}\right)\right)$ is a real, scalar-valued function, bounded away from zero and finite on $\Omega \backslash\left(O_{\infty} \cup O_{0}\right)$ and where $\sigma_{0} \in C^{0}(\Omega, \operatorname{Mat}(\mathbb{R}, n))$ is symmetric, positive definite.

We will prove that the shape and locations of the perfectly conducting and insulating inclusions and the conductivity $\sigma$ outside of the inclusions are determined from knowledge of the boundary voltage $f, \sigma_{0}$ and

$$
a=\sqrt{\sigma_{0}^{-1} J \cdot J}=|J|_{\sigma_{0}^{-1}} \text { in } \Omega
$$

where $J$ is the current density vector field generated by imposing the voltage $f$ at $\partial \Omega$. To formulate our results, we first need to extend the notion of admissibility.

Definition 4.1. A triplet of functions $\left(f, \sigma_{0}, a\right) \in H^{1 / 2}(\partial \Omega) \times C^{0}(\Omega, \operatorname{Mat}(\mathbb{R}, n)) \times L^{\infty}(\Omega)$ is called admissible if there exists a matrix valued function $\sigma$ satisfying (28) and a divergence free vector field $J \in\left(L^{\infty}(\Omega)\right)^{n}$ such that the following three statements hold.

$$
a=|J|_{\sigma_{0}^{-1}} \text { in } \Omega
$$

(ii) The vector field $J$ satisfies

$$
J= \begin{cases}-\sigma \nabla u & \text { in } \Omega \backslash\left(O_{\infty} \cup O_{0}\right) \\ 0 & \text { in } O_{0}\end{cases}
$$

where $u$ is the corresponding solution of (27).

(iii) The set of zeros of a can be decomposed as follows

$$
\{x \in \Omega: a(x)=0\} \cap\left(\Omega \backslash \bar{O}_{\infty}\right)=O_{0} \cup \bar{O}_{s} \cup \Gamma,
$$


where $O_{s}$ is an open set (possibly empty), $\Gamma$ is a Lebesgue-negligible set, and $\bar{\Gamma}$ has empty interior.

We are now ready to state our main uniqueness results.

Theorem 4.2. Let $\Omega \subset \mathbb{R}^{n}, n \geq 2$, be a domain with connected Lipschitz boundary and let $\left(f, \sigma_{0}, a\right) \in H^{1 / 2}(\partial \Omega) \times C^{0}(\Omega, \operatorname{Mat}(\mathbb{R}, n)) \times L^{\infty}(\Omega)$ be an admissible triplet generated by an unknown conductivity $\sigma$. Define $\phi(x, \xi)=a(x)|\xi|_{\sigma_{0}}$ on $\Omega \backslash \bar{S}$.

Then the potential u, solving (27), is a minimizer of the problem

$$
u=\operatorname{argmin}\left\{\int_{\Omega}|D v|_{\phi}: v \in B V(\Omega \backslash \bar{S}) \text { and }\left.v\right|_{\partial \Omega}=f\right\}
$$

and, if $\bar{u}$ is another minimizer of the above problem, then $\bar{u}=u$ in $\Omega \backslash\{|J|=0\}$.

Moreover the zero-sets of $a$ and $|\nabla u|$ can be decomposed as follows

$$
\{x \in \Omega, a(x)=0\} \cup\{x \in \Omega: \nabla u=0\}=: Z \cup \Gamma,
$$

where $\Gamma$ has measure zero and $Z=O_{\infty} \cup O_{0} \cup O_{s}$ is open.

Consequently

$$
\sigma=\frac{a}{|\nabla u|_{\sigma_{0}}} \sigma_{0} \in C^{\alpha}(\Omega \backslash \bar{Z})
$$

is the unique conductivity generating the admissible data triplet $\left(f, \sigma_{0}, a\right)$.

Remark The above theorem allows us to identify the potential $u$ and the conductivity $\sigma$ outside the open set $Z=O_{\infty} \cup O_{0} \cup S$. To determine if an open connected component $O$ of $Z$ is a perfectly conducting inclusion, an insulating inclusion, or a singular inclusion we proceed as follows:

- If $\nabla u \equiv 0$ in $O$ and $a(x) \neq 0$ for some $x \in O$, then $O$ is a perfectly conducting inclusion.

- If $a \equiv 0$ in $O$ and $u \not \equiv$ constant on $\partial O$, then $O$ is an insulating inclusion.

- If $a \equiv 0$ in $O, u=$ constant on $\partial O$, and $a$ is not $C^{\alpha}$ at $x$ for some $x \in O$, then $O$ is either an insulating inclusion or a perfectly conducting inclusion.

- If $a \equiv 0, u=$ constant on $\partial O$, and $a \in C^{\alpha}(\partial O)$, then the knowledge of the magnitude of the current $\left(f, \sigma_{0}, a\right)$ is not enough to determine the type of the inclusion $O$.

Proof. Suppose that $\bar{u} \in B V_{l o c}(\Omega \backslash \bar{S})$. First note that for every $x \in \Omega \backslash\left(\overline{O_{\infty} \cup O_{0}}\right)$ there exists $\epsilon>0$ such that $B(x, 2 \epsilon) \subset \Omega$ and

$$
\int_{B(x, \epsilon)} h\left(x, v^{\bar{u}}\right)|D \bar{u}| \geq-\int_{B(x, \epsilon)} J \cdot v^{\bar{u}}|D \bar{u}|,
$$


where $J$ is the current density vector field described in definition (4.1). Therefore

$$
h\left(x, v^{\bar{u}}\right) \geq-J \cdot v^{\bar{u}}, \quad|D \bar{u}|-a . e \text {. in } \Omega \backslash\left(\overline{O_{\infty} \cup O_{0}}\right) .
$$

Thus, on using Lemma 3.1 and the fact that the current density is divergence-free away from the inclusions we have

$$
\begin{aligned}
\int_{\Omega \backslash \overline{\left(O_{0} \cup O_{\infty}\right)}}|D \bar{u}|_{\varphi} & =\int_{\Omega \backslash \overline{\left(O_{0} \cup O_{\infty}\right)}} h\left(x, v^{\bar{u}}\right)|D \bar{u}| \\
& \geq-\int_{\Omega \backslash \overline{\left(O_{0} \cup O_{\infty}\right)}} J \cdot v^{\bar{u}}|D \bar{u}| \\
& =-\int_{\Omega \backslash \overline{\left(O_{0} \cup O_{\infty}\right)}} J \cdot D \bar{u} \\
& =-\int_{\partial \Omega \backslash \overline{\left(O_{0} \cup O_{\infty}\right)}} J \cdot \nu f d \mathcal{H}^{n-1} \\
& =\int_{\Omega \backslash \overline{\left(O_{0} \cup O_{\infty}\right)}}|D u|_{\varphi} .
\end{aligned}
$$

Hence

$$
h\left(x, v^{\bar{u}}\right)=-J \cdot v^{\bar{u}}, \quad|D \bar{u}|-a . e . \text { in } \Omega \backslash \overline{\left(O_{0} \cup O_{\infty}\right)}
$$

Then again, since $a$ is continuous in $\Omega \backslash\left(\overline{O_{0} \cup O_{\infty}}\right)$ equation (21) gives

$$
h\left(x, v^{\bar{u}}\right)=a(x)\left(\sum_{i, j=1}^{n} \sigma_{0}^{i j} v_{i}^{\bar{u}} v_{j}^{\bar{u}}\right)^{1 / 2}|D \bar{u}|-a . e . \text { in } \Omega \backslash\left(\overline{O_{0} \cup O_{\infty}}\right) .
$$

But then, on $\Omega \backslash\left(\overline{O_{0} \cup O_{\infty}}\right)$, we have

$$
\begin{aligned}
h\left(x, v^{\bar{u}}\right) & =a(x)\left(\sum_{i, j=1}^{n} \sigma_{0}^{i j} v_{i}^{\bar{u}} v_{j}^{\bar{u}}\right)^{1 / 2} \\
& =c(x)|\nabla u|_{\sigma_{0}}\left|v^{\bar{u}}\right|_{\sigma_{0}} \\
& \geq c(x)\left|\left(\nabla u, v^{\bar{u}}\right)_{\sigma_{0}}\right| \\
& \geq \sigma \nabla u \cdot v^{\bar{u}} \\
& =-J \cdot v^{\bar{u}} .
\end{aligned}
$$

Thus it follows from (32) that

$$
\frac{J}{|J|}=\frac{\nabla \bar{u}}{|\nabla \bar{u}|}=v^{\bar{u}}, \quad|D \bar{u}|-\text { a.e. in } \Omega \backslash \overline{\left(O_{0} \cup O_{\infty}\right)} .
$$

It follows from an argument similar to that of Theorem 3.5 in [28 that $u=\bar{u}$ a.e. in $\Omega$. 


\section{$5 \quad$ Geometrical Properties of Equipotential Sets}

In this section we prove the area-minimizing property of the equipotential sets $u^{-1}(\lambda):=$ $\bar{\Omega} \cap\{x ; u(x)=\lambda\}$ for solutions $u(x)$ of the equation (27). This generalizes results in [33] and [29]. The basic argument goes back to $([10])$. We shall need the following lemmas, which require the generalized coarea formula (25).

Define the functional $\mathcal{F}$ by

$$
\mathcal{F}[u]:=\int_{\Omega}|D v|_{\phi}
$$

for the positive definite, matrix-valued $\sigma_{0} \in C^{1, \alpha}(\bar{\Omega}, \operatorname{Mat}(\mathbb{R}, n))$. As well, for a given function $w$ on $\Omega$, we introduce the notation

$$
w_{\lambda, \epsilon}:=\frac{1}{\epsilon} \min \{\epsilon, \max w-\lambda, 0\}
$$

for fixed $\lambda$ and $\epsilon>0$. For functions $w$ on $\partial \Omega, w_{\lambda, \epsilon}$ denotes the trace at the boundary of such functions.

Lemma 5.1. Let $a \in \operatorname{Lip}(\Omega)$, fix $\lambda \in \mathbb{R}$ and let $\epsilon>0$. Then if

$$
u \in \underset{\left.v\right|_{\partial \Omega}=f}{\operatorname{argmin}}\left\{\int_{\Omega}|D v|_{\phi} ; \quad v \in W_{+}^{1,1}(\Omega)\right\}
$$

Then also

$$
u_{\lambda, \epsilon} \in \underset{\left.v\right|_{\partial \Omega}=f_{\lambda, \epsilon}}{\operatorname{argmin}}\left\{\int_{\Omega}|D v|_{\phi} ; v \in W_{+}^{1,1}(\Omega)\right\}
$$

where $u_{\lambda, \epsilon}=\frac{1}{\epsilon} \min \{\epsilon, \max u-\lambda, 0\}$.

Proof. For $u \in W_{+}^{1,1}(\Omega)$ we have $v^{u}=\frac{\nabla u}{|\nabla u|} \mu-$ a.e. Set $u_{+}=\max \{u-\lambda, 0\}$ and $u_{-}=u-u_{+}$. Then since $a$ and $\sigma_{0}$ are continuous we have, with $\phi(x, \xi)=a|\xi|_{\sigma_{0}}$, that

$$
\int_{\Omega}|D u|_{\phi}=\int_{\Omega} a(x)\left(\sigma_{0} v^{u} \cdot v^{u}\right)|\nabla u|=\mathcal{F}[u]
$$

Then again, by (25), we have

$$
\begin{aligned}
\mathcal{F}[u] & =\int_{t<\lambda} \int_{u^{-1}(t)} a(x) d \mathcal{H}^{n-1}(x) d t+\int_{t>\lambda} \int_{u^{-1}(t)} a(x) d \mathcal{H}^{n-1}(x) d t \\
& =\int_{t \in \mathbb{R}}\left\{\int_{u_{+}^{-1}(t)} a(x) \mathcal{H}^{n-1}(x)+\int_{u_{-}^{-1}(t)} a(x) d \mathcal{H}^{n-1}(x)\right\} d t \\
& =\mathcal{F}\left[u_{+}\right]+\mathcal{F}\left[u_{-}\right]
\end{aligned}
$$


Suppose that $u \in \operatorname{argmin}\left\{\mathcal{F}[v] ; v \in W_{+}^{1,1}(\Omega)\right\}$, whereby one has with a test function $w \in$ $\left.v\right|_{\partial \Omega}=f$

$\operatorname{Lip}(\Omega)$ chosen such that $\left.w\right|_{\partial \Omega}=0$ that

$$
\begin{aligned}
\mathcal{F}\left[u_{+}\right] & =\mathcal{F}[u]-\mathcal{F}\left[u_{-}\right] \\
& \leq \mathcal{F}[u+w]-\mathcal{F}\left[u_{-}\right] \\
& \leq \mathcal{F}\left[u_{+}+u_{-}-u_{-}+w\right]
\end{aligned}
$$

so that $u_{+} \in \operatorname{argmin}\left\{\mathcal{F}[v] ; v \in W_{+}^{1,1}(\Omega)\right\}$ and, likewise $u_{-} \in \operatorname{argmin}\left\{\mathcal{F}[v] ; v \in W_{+}^{1,1}(\Omega)\right\}$ $\left.v\right|_{\partial \Omega}=f_{+}$
follows mutatis mutandis. The lemma is immediate from two applications of this fact.

Lemma 5.2. Let $a \in \operatorname{Lip}(\Omega)$ and $u \in \operatorname{Lip}(\Omega)$ be such that

$$
\{x ;|\nabla u|=0\}=Z \cup L
$$

where $Z$ is open, $a(\bar{Z})=0, \mu(L)=0$ and

$$
\frac{a \nabla u}{|\nabla u|} \in W^{1,1}(\Omega / \bar{Z})
$$

Then

$$
\lim _{\epsilon \downarrow 0} \mathcal{F}\left[u_{\lambda, \epsilon}\right]=\int_{u^{-1}(\lambda)} a(x) d \mathcal{H}^{n-1}(x)
$$

holds for almost every $\lambda \in \mathbb{R}$.

Proof. The proof is similar to that of Lemma 4.3 in [29].

We consider the functional

$$
\mathcal{A}(\Sigma)=\int_{\Sigma}|J|_{\sigma_{0}^{-1}} d S
$$

for $\Sigma \subset \Omega$ and $d S$ the induced Euclidean surface measure on the $n-1$ dimensional subset $\Sigma$. We remark that when $\Omega$ is equipped with the data dependent metric $g_{i j}=\left(\left|\sigma_{0}\right||J|_{\sigma_{0}^{-1}}^{2}\right)^{\frac{1}{n-1}}\left(\sigma_{0}^{-1}\right)_{i j}$ discussed in Proposition 2.4 that the invariant volume form $\sqrt{|g|} d x^{1} \wedge \cdots \wedge d x^{n}$ on $\Omega$ induces the invariant volume form $|J|_{\sigma_{0}^{-1}} d S(x)$ on smooth $n-1$ dimensional hypersurfaces $\Sigma$. For this reason we refer to (35) as an area functional.

We are ready to establish the main result of this section, which says that equipotential hypersurfaces of solutions to (27) are minimizers of the area functional (35).

Theorem 5.3. Let $\Omega \subset \mathbb{R}^{n}, n \geq 2$, be a domain with connected Lipschitz boundary and let $\left(f, \sigma_{0}, a\right) \in C^{2, \delta}(\partial \Omega) \times C^{1, \delta}(\Omega, \operatorname{Mat}(\mathbb{R}, n)) \times L^{2}(\bar{\Omega})$ be an admissible triplet generated by an unknown conductivity $\sigma \in C^{1, \delta}\left(\Omega \backslash\left(O_{\infty} \cup O_{0}\right)\right.$ with $\delta \in(0,1)$. Let $v \in C^{2, \delta}(\bar{\Omega})$ satisfy $\left.v\right|_{\partial \Omega}=f$ and

$$
\{x ;|\nabla v|=0\}=Z_{v} \cap L_{v}, \quad a\left(\overline{Z_{v}}\right)=0
$$

for $Z_{v}$ open and $\mu\left(L_{v}\right)=0$. 
Then, when $u$ is the solution to the BVP (27) we have

$$
\mathcal{A}\left(u^{-1}(\lambda)\right) \leq \mathcal{A}\left(v^{-1}(\lambda)\right)
$$

for almost all $\lambda \in \mathbb{R}$.

Proof. Notice that when $\lambda \notin R a n g e(u)$, the result is immediate. Otherwise since, under these hypotheses on the conductivity, $u$ satisfies the maximum principle and, by assumption $u$ and $v$ agree on $\partial \Omega, \operatorname{Range}(u) \subset \operatorname{Range}(v)$. If $\lambda \in \operatorname{Range}(u) /\left\{u(\bar{Z}) \cup v\left(\overline{Z_{v}}\right)\right\}$ is arbitrary,

$u(\bar{Z}) \cup v\left(\overline{Z_{v}}\right)$ is at most countable, and since $\nabla u$ and $\nabla v$ are both non-zero away from $\bar{Z}$ and $\overline{Z_{v}}$ respectively, $u^{-1}(\lambda)$ and $v^{-1}(\lambda)$ are $C^{2}$-smooth oriented hypersurfaces whereon the Hausdorff measure coincides with the standard Lebesgue measure. Thus $\mathcal{H}^{n}\left(u^{-1}(\lambda)\right)=0$ for a.e. $\lambda$ and, by assumption $\mathcal{H}^{n-1}\left(u^{-1}(\lambda) \cap \partial \Omega\right)=0$ for a.e. $\lambda$ since $u$ extends continuously to the boundary, which has finite Hausdorff measure.

Since $u$ and $v$ agree on the boundary $\left.u_{\lambda, \epsilon}\right|_{\partial \Omega}=\left.v_{\lambda, \epsilon}\right|_{\partial \Omega}$ as well. But then by Theorem 4.2 we have

$$
\mathcal{F}\left[u_{\lambda, \epsilon}\right] \leq \mathcal{F}\left[v_{\lambda, \epsilon}\right]
$$

The result then follows from (34).

\section{Appendix: Perfectly conductive and insulating in- clusions}

The results presented in this appendix give a precise definition, by a limiting procedure, of potentials corresponding to conductivities that can vanish or be infinite in certain regions. They are slight generalization of the ones in [7] to include both perfectly conductive and insulating inclusions.

Let $O_{\infty}=\cup_{j=1}^{\infty} O_{\infty}^{j}$ be an open subset of $\Omega$ with $\bar{O}_{\infty} \subset \Omega$ to model the union of the connected components $O_{\infty}^{j}(j=1,2, \ldots)$ of perfectly conductive inclusions, and let $O_{0}$ be an open subset of $\Omega$ with $\bar{O}_{0} \subset \Omega$ to model the union of all connected insulating inclusions. Let $\chi_{O_{\infty}}$ and $\chi_{O_{0}}$ be their corresponding characteristic function. We assume that $\overline{O_{\infty}} \cap \overline{O_{0}}=\emptyset$, $\Omega \backslash \overline{O_{\infty} \cup O_{0}}$ is connected, and that the boundaries $\partial O_{\infty}, \partial O_{0}$ are piecewise $C^{1, \alpha}$ for $\alpha>$ 0 . Let $\sigma_{1} \in C^{\alpha}\left(O_{\infty}, \operatorname{Mat}(\mathbb{R}, n)\right)$, and $\sigma \in C^{\alpha}\left(\Omega \backslash \overline{O_{\infty} \cup O_{0}}, \operatorname{Mat}(\mathbb{R}, n)\right)$ be matrix-valued functions such that

$$
m|\xi|^{2} \leq \sigma^{i j} \xi_{i} \xi_{j} \leq M|\xi|^{2}, \quad m|\xi|^{2} \leq \sigma_{1}^{i j} \xi_{i} \xi_{j} \leq M|\xi|^{2}
$$

for constants $0<m, M<\infty$ and all $\xi \in \mathbb{R}^{n}$.

For each $0<k<1$ consider the conductivity problem

$$
\nabla \cdot\left(\chi_{U}\left(\frac{1}{k} \sigma_{1}-\sigma\right)+\sigma\right) \nabla u=0, \quad \frac{\partial u}{\partial \nu}=0 \text { on } \partial O_{0}, \quad \text { and }\left.\quad u\right|_{\partial \Omega}=f .
$$


The condition on $\partial O_{0}$ ensures that $O_{0}$ is insulating. It is well known that the problem (37) has a unique solution $u_{k} \in H^{1}(\Omega)$ which also solves

$$
\begin{cases}\nabla \cdot \sigma \nabla u_{k}=0, & \text { in } \Omega \backslash \overline{O_{\infty} \cup O_{0}} \\ \nabla \cdot \sigma_{1} \nabla u_{k}=0, & \text { in } O_{\infty}, \\ \left.u_{k}\right|_{+}=\left.u_{k}\right|_{-}, & \text {on } \partial O_{\infty}, \\ \left.\left(\frac{1}{k} \sigma_{1} \nabla u_{k}\right) \cdot \nu\right|_{-}=\left.\left(\sigma \nabla u_{k}\right) \cdot \nu\right|_{+}, & \text {on } \partial O_{\infty}, \\ \left.\frac{\partial u_{k}}{\partial \nu}\right|_{+}=0, & \text { on } \partial O_{0}, \\ \left.u_{k}\right|_{\partial \Omega}=f . & \end{cases}
$$

Moreover, the energy functional

$$
I_{k}[v]=\frac{1}{2 k} \int_{O_{\infty}}|\nabla v|_{\sigma_{1}}^{2} d x+\frac{1}{2} \int_{\Omega \backslash \overline{U \cup V}}|\nabla v|_{\sigma}^{2} d x
$$

has a unique minimizer over the maps in $H^{1}(\Omega)$ with trace $f$ at $\partial \Omega$ which is the unique solution $u_{k}$ of (38).

We shall show below that the limiting solution (with $k \rightarrow 0$ ) solves

$$
\begin{cases}\nabla \cdot \sigma \nabla u_{0}=0, & \text { in } \Omega \backslash \overline{O_{\infty} \cup O_{0}} \\ \nabla u_{0}=0, & \text { in } O_{\infty} \\ \left.u_{0}\right|_{+}=\left.u_{0}\right|_{-}, & \text {on } \partial O_{\infty} \\ \left.\int_{\partial O_{\infty}^{j}}(\sigma \nabla u) \cdot \nu\right|_{+} d s=0, & j=1,2, \ldots \\ \frac{\partial u_{0}}{\partial \nu_{+}}=0, & \text { on } \partial O_{0} \\ \left.u_{0}\right|_{\partial \Omega}=f, & \end{cases}
$$

By elliptic regularity $u_{0} \in C^{1, \alpha}\left(\Omega \backslash O_{\infty} \cup O_{0}\right)$ and for any $C^{1, \alpha}$ boundary portion $T$ of $\partial\left(O_{\infty} \cup\right.$ $\left.O_{0}\right), u_{0} \in C^{1, \alpha}\left(\left(\Omega \backslash\left(O_{\infty} \cup O_{0}\right)\right) \cup T\right)$.

Proposition 6.1. The problem (40) has a unique solution in $H^{1}(\Omega)$. This solution is the unique minimizer of the functional

$$
I_{0}[v]=\frac{1}{2} \int_{\Omega \backslash \overline{O_{\infty} \cup O_{0}}}|\nabla v|_{\sigma}^{2} d x
$$

over the set $A_{0}:=\left\{u \in H^{1}\left(\Omega \backslash \bar{O}_{0}\right) ;\left.u\right|_{\partial \Omega}=f, \nabla u=0\right.$ in $\left.O_{\infty}\right\}$.

Proof: Note that $A_{0}$ is weakly closed in $H^{1}\left(\Omega \backslash \bar{O}_{0}\right)$. The functional $I_{0}$ is lower semicontinuous and strictly convex and, as a consequence, has a unique minimizer $u_{0}^{*}$ in $A_{0}$.

First we show that $u_{0}^{*}$ is a solution of the BVP (40). Since $u_{0}^{*}$ minimizes (41), we have

$$
0=\int_{\Omega \backslash \overline{O_{0} \cup O_{\infty}}} \sigma \nabla u_{0}^{*} \cdot \nabla \phi d x
$$

for all $\phi \in H^{1}\left(\Omega \backslash \overline{O_{0}}\right)$, with $\left.\phi\right|_{\partial \Omega}=0$, and $\nabla \phi=0$ in $O_{\infty}$. In particular, if $\phi \in H_{0}^{1}\left(\Omega \backslash \bar{O}_{0}\right)$, we get $\int_{\Omega \backslash O_{\infty} \cup O_{0}}\left(\nabla \cdot \sigma \nabla u_{0}^{*}\right) \phi d x=0$ and thus $u_{0}^{*}$ solves the conductivity equation in (40). If 
we choose $\phi \in H^{1}\left(\Omega \backslash \bar{O}_{0}\right)$, with $\left.\phi\right|_{\partial \Omega}=0$, and $\phi \equiv 0$ in $O_{\infty}$, from Green's formula applied to (42), we get $\left.\int_{\partial O_{0}}\left(\sigma \nabla u_{0}^{*}\right) \cdot \nu\right|_{+} \phi=0,\left.\forall \phi\right|_{\partial O_{0}} \in H^{1 / 2}\left(\partial O_{0}\right)$, or equivalently, $\left.\frac{\partial u_{0}^{*}}{\partial \nu}\right|_{\partial O_{0}}=0$. If we choose $\phi_{j} \in H_{0}^{1}\left(\Omega \backslash \bar{O}_{0}\right)$ with $\phi_{j} \equiv 1$ in the connected component $O_{\infty}^{j}$ of $O_{\infty}$ and $\phi_{j} \equiv 0$ in $O_{\infty} \backslash O_{\infty}^{j}$, from Green's formula applied to (42) we obtain $\left.\int_{\partial O_{\infty}^{j}}\left(\sigma \nabla u_{0}^{*}\right) \cdot \nu\right|_{+}=0$.

Next we show that the equation (40) has a unique solution and, consequently, $u_{0}^{*}=$ $\left.u_{0}\right|_{\Omega \backslash \bar{O}_{0}}$. Assume that $u^{1}$ and $u^{2}$ are two solutions and let $u=u_{2}-u_{1}$, then $\left.u\right|_{\partial \Omega}=0$ and

$$
\begin{aligned}
0=- & \int_{\Omega \backslash \overline{O_{\infty} \cup O_{0}}}(\nabla \cdot \sigma \nabla u) u d x=-\int_{\partial \Omega}(\sigma \nabla u) \cdot \nu u d s+\left.\int_{\partial O_{0}}(\sigma \nabla u) \cdot \nu\right|_{+} u d s \\
& +\left.\int_{\partial O_{\infty}}(\sigma \nabla u) \cdot \nu\right|_{+} u d s+\int_{\Omega \backslash \overline{O_{\infty} \cup O_{0}}}|\nabla u|_{\sigma}^{2} d x=\int_{\Omega \backslash \overline{O_{\infty} \cup O_{0}}}|\nabla u|_{\sigma}^{2} d x
\end{aligned}
$$

Thus $|\nabla u| \equiv 0$ in $\Omega \backslash \bar{O}_{0}$. Since $\Omega \backslash \bar{O}_{0}$ is connected and $u=0$ at the boundary, we conclude uniqueness of the solution of the equations (40).

Theorem 6.2. Let $u_{k}$ and $u_{0}$ be the unique solution of (38) respectively (40) in $H^{1}(\Omega)$. Then $u_{k} \rightarrow u$ and, consequently, $I_{k}\left[u_{k}\right] \stackrel{k \downarrow 0^{+}}{\longrightarrow} I_{0}[u]$.

Proof: We show first that $\left\{u_{k}\right\}$ is bounded in $H^{1}(\Omega)$ uniformly in $k \in(0,1)$. Since $1 / k>1$, we have

$$
\begin{array}{r}
\frac{\lambda}{2}\left\|\nabla u_{k}\right\|_{L^{2}\left(\Omega \backslash \bar{O}_{0}\right)}^{2} \leq \frac{1}{2} \int_{\Omega \backslash \bar{O}_{\infty} \cup O_{0}}\left|\nabla u_{k}\right|_{\sigma}^{2} d x+\frac{1}{2 k} \int_{O_{\infty}}\left|\nabla u_{k}\right|_{\sigma_{1}}^{2} d x \\
\leq I_{k}\left[u_{k}\right] \leq I_{k}\left[u_{0}\right] \leq \frac{\Lambda}{2}\|\nabla u\|_{L^{2}\left(\Omega \backslash \bar{O}_{0}\right)}^{2},
\end{array}
$$

or

$$
\left\|\nabla u_{k}\right\|_{L^{2}\left(\Omega \backslash \bar{O}_{0}\right)}^{2} \leq \frac{\Lambda}{\lambda}\|\nabla u\|_{L^{2}\left(\Omega \backslash \bar{O}_{0}\right)}^{2}
$$

From (45) and the fact that $\left.u_{k}\right|_{\partial \Omega}=f$, we see that $\left\{u_{k}\right\}$ is uniformly bounded in $H^{1}\left(\Omega \backslash O_{0}\right)$ and hence weakly compact. Therefore, there is a subsequence $u_{k} \rightarrow u^{*}$ in $H^{1}\left(\Omega \backslash O_{0}\right)$, for some $u^{*}$ with trace $f$ at $\partial \Omega$.

We will show next that $u^{*}$ satisfies the equations (40), and therefore $u^{*}=u_{0}$ on $\Omega \backslash O_{0}$. By the uniqueness of solutions of (40) we also conclude that the whole sequence converges to $u$.

Since $u_{k} \rightarrow u^{*}$ we have that $0=\int_{\Omega \backslash \overline{O_{0} \cup O_{\infty}}} \sigma \nabla u_{k} \cdot \nabla \phi d x \rightarrow \int_{\Omega \backslash \overline{O_{\infty} \cup O_{0}}} \sigma \nabla u^{*} \cdot \nabla \phi d x$, for all $\phi \in C_{0}^{\infty}\left(\Omega \backslash \overline{O_{\infty} \cup O_{0}}\right)$. Therefore $\nabla \cdot \sigma \nabla u^{*}=0$ in $\Omega \backslash \overline{O_{\infty} \cup O_{0}}$. Further, since $u_{k}$ minimizes $I\left[u_{k}\right]$ we must have $\nabla u^{*}=0$ in $O_{\infty}$. To check the boundary conditions, note that, for all $\phi \in C_{0}^{\infty}(\Omega)$ with $\phi \equiv 0$ in $O_{\infty}$, we have $\left.\int_{\partial O_{0}}\left(\sigma \nabla u_{k}\right) \cdot \nu\right|_{+} \phi d s=0$. Using the fact that $\phi$ were arbitrary, by taking the weak limit in $k \rightarrow 0$, we get $\left.\frac{\partial u^{*}}{\partial \nu}\right|_{+}=0$ on $\partial V$. A similar argument applied to $\phi \in C_{0}^{\infty}(\Omega)$ with $\phi \equiv 0$ in $O_{0}, \phi \equiv 1$ in $O_{\infty}^{j}$, and $\phi \equiv 0$ in $O_{\infty} \backslash O_{\infty}^{j}$, also shows that $\left.\int_{\partial O_{\infty}^{j}}\left(\sigma \nabla u^{*}\right) \cdot \nu\right|_{+} \phi d s=0$. Hence $u^{*}$ is the unique solution of the equation (40) on $\Omega \backslash \bar{O}_{0}$. Thus $u_{k}$ converges weakly to the solution $u_{0}$ of (40) in $\Omega \backslash \bar{O}_{0}$. 


\section{Conclusions}

We have considered the reconstruction of an anisotropic conductivity conformal to a known $\sigma_{0}$ when one has knowledge of the internal functional $\sqrt{\sigma_{0}^{-1} J \cdot J}$. Such data can be obtained by a novel combination of Current Density and Diffusion Tensor measurements. We have identified a variational problem defined in terms of the measured data and shown how to calculate the conformal factor from its unique solution. Further, we have presented a solution of the problem which allows for regions of infinite or zero conductivity, and which does not explicitly use Ohm's law in such regions. We also proved that the equipotential sets minimize the area functional corresponding to a Riemannian metric defined from the measured data.

\section{Acknowledgements}

The authors wish to kindly thank Mike Joy, Weijing Ma, and Nahla Elsaid for insightful discussions on DTI and the relation between the conductivity and diffusion tensors.

\section{References}

[1] G. Alberti, A Lusin type theorem for gradients, J. Functional Analysis, 100 (1991), pp. $110-118$.

[2] M. Amar and G. Bellettini, A notion of total variation depending on a metric with discontinuous coefficients, Annales de l'I.H.P., 11 (1994), pp. 91-133.

[3] H. Ammari, E. Bonnetier, Y. Capdeboscq, M. Tanter, And M. Fink, Electrical impedance tomography by elastic deformation, SIAM J. Appl. Math., 68 (2008), pp. $1557-1573$.

[4] H. Ammari, J. Garnier, H. Kang, W.-K. Park, and K. Solna, Imaging schemes for perfectly conducting cracks, SIAM J. Appl. Math., 71 (2011), pp. 68-91.

[5] G. Anzellotti, Traces of bounded vector fields and the divergence theorem, Preprint, University of Trento, (1983).

[6] G. BAL, Hybrid Inverse Problems and Internal Functionals, in Inside Out II, G. Uhlmann, ed., no. 60 in MSRI Publications, Cambridge University Press, 2012.

[7] E. S. BAO, Y. Y. LI, AND B. Yin, Gradient estimates for the perfect conductivity problem, Arch. Rational Mech. Anal., 193 (2009), pp. 195-226.

[8] J. Belliveau, A. Dale, J. George, D. Tuch, and V. Wedeen, Conductivity tensor mapping of the human brain using diffusion tensor MRI, Proc. Natl. Acad. Sci., 20 (1998), pp. 11697-11701. 
[9] L. Bers, F. John, And M. Schechter, Partial Differential Equations, Wiley \& Sons, New York, 1964.

[10] E. Bombieri, E. D. Giorgi, and E. Giusti, Minimal cones and the Bernstein problem, Invent. Math., 7 (1969), pp. 243-268.

[11] L. C. Evans and R. Gariepy, Measure Theory and Fine Propoerties of Functions, CRC Press, 1992.

[12] H. Federer, Geometric Measure Theory, Springer, 1996.

[13] D. D. S. Ferreira, C. Kenig, M. Salo, and G. Uhlmann, Limiting Carleman weights and anisotropic inverse problems, Invent. Math., 178 (2009), pp. 119-171.

[14] B. Gebauer and O. Scherzer, Impedance-acoustic tomography, SiAM J. Appl. Math., 69 (2008), pp. 565-576.

[15] E. Giusti, Minimal Surfaces and Functions of Bounded Variation, Birkhäuser, 1984.

[16] D. Isaacson, Distinguishability of conductivities by electrical-current computed tomography, IEEE Trans, Med. Imag, 5 (1986), pp. 91-95.

[17] M. Joy, G. Scott, and M. Henkelman, In vivo detection of applied electric currents by magnetic resonance imaging by magnetic resonance imaging,, Magenetic Resonance Imaging, 7 (1989), pp. 89-94.

[18] C. Kenig, M. Salo, and G. Uhlmann, Reconstructions from boundary measurements on admissible manifolds, Inverse Problems and Imaging, 5 (2011), pp. 859-877.

[19] S. Kim, O. Kwon, J. SEo, And J. Yoon, On a nonlinear partial differential equation arising in magnetic resonance electrical impedance tomography, SIAM J. Math. Anal., (2002), pp. 511-526.

[20] P. Kuchment, Mathematics of Hybrid Imaging. A Brief Review, in The Mathematical Legacy of Leon Ehrenpreis, I. Sabadini and D. Struppa, eds., Springer, 2012.

[21] P. Kuchment and L. Kunyansky, Synthetic focusing in ultrasound modulated tomography, Inverse Problems and Imaging, 4 (2010).

[22] A. Liseno And R. PierRi, Imaging perfectly conducting objects as support of induced currents: Kirchhoff approximation and frequency diversity, Journal of the Optical Society of America A, 19 (2002), pp. 1308-1318.

[23] W. Ma, A. Nachman, N. Elsaid, M. Joy, and T. DeMonte, Anisotropic impedance imaging using diffusion tensor and current density measurements, Preprint, (2013). 
[24] F. Maggi, Sets of Finite Perimeter and Geometric Variational Problems, Cambridge University Press, 2012.

[25] N. MANDACHE, Exponential instability in an inverse problem for the Schrödinger equation, Inverse Problems, 17 (2001), pp. 1435-1444.

[26] O. Martio, Counterexamples for unique continuation, Manuscripta Math., 60 (1988), p. 21.

[27] W. Massey, Algebraic Topology: An Introduction, Springer-Verlag, 1977.

[28] A. Moradifam, A. Nachman, And A. TAmasan, Uniqueness of minimizers of weighted least gradient problems arising in conductivity imaging, In Preparation.

[29] — Conductivity imaging from one interior measurement in the presence of perfectly conducting and insulating inclusions, SIAM J. Math. Anal, (to appear).

[30] F. Morgan, Geometric Measure Theory, Academic Press, 2000.

[31] A. Nachman, A. Tamasan, and A. Timonov, Conductivity imaging with a single measurement of boundary and interior data, Inverse Problems, 23 (2007), pp. 2551-2563.

[32] — Recovering the conductivity from a single measurement of interior data, Inverse Problems, 23 (2009).

[33] _ Reconstruction of planar conductivities in subdomains from incomplete data, SIAM J. Appl. Math., 70 (2010), pp. 3342-3362.

[34] - Current density impedance imaging, in Tomography and Inverse Transport Theory, G. Bal, D. Finch, P. Kuchment, J. Schotland, P. Stefanov, and G. Uhlmann, eds., no. 559 in Contemp. Math., American Mathematical Society, 2011.

[35] P. Nicholson, Specific impedance of cerebral white matter, Experimental Neurology, 13 (1965), pp. 386-401.

[36] A. PLIŚ, On non-uniquness in cauchy problem for an elliptic second order differantial equation, Bull. Acad. Pol. Sci., S. Mat., XI (1963), pp. 95-100.

[37] B. Rотн, The electrical conductivity of tissues, The Biomedical Engineering Handbook; CRC Press, (2000).

[38] G. Scott, M. Joy, R. Armstrong, and R. Henkelman, Measurement of nonuniform current density by magnetic resonance, IEEE Trans, Med. Imag, (1991), pp. 362374.

[39] J. K. Seo, D. H. Kim, J. Lee, O. I. Kwon, S. Z. K. Sajib, and E. J. Woo, Electrical tissue property imaging using MRI at dc and Larmor frequency, Inverse Problems, 28 (2012). 
[40] G. Uhlmann, Electrical impedance tomography and Calderón's problem, Inverse Problems, 25 (2009).

[41] H. Zhang And L. V. Wang, Acousto-electric tomography, Photons Plus Ultrasound: Imaging and Sensing (A. A. Oraevsky and L. V. Wang, Eds.), 5320 (2004), pp. 145-149. 